24. Shimada, M., and Langman, J.: Cell proliferation, migration and differentiation in the cerebral cortex of the golden hamster. J. Comp. Neurol., 139; 227 (1970).

25. Shimada, M., and Nakamura, T.: Time of neuron origin in mouse hypothalamic nuclei. Exp. Neurol., 41: 163 (1973).

26. Taber-Pierce, E.: Histogenesis of the nuclei griseum pontis corporis pontobulbaris and reticularis tegmenti pontis (Bechterew) in the mouse: An autoradiographic study. J. Comp. Neurol., 126: 219 (1966).

27. Taber-Pierce, E.: Histogenesis of the dorsal and ventral cochlear nuclei in the mouse: An autoradiographic study. J. Comp. Neurol., 131: 27 (1967).

28. Warkany, J., Monroe, B. B., and Sutherland, B. S.: Intrauterine growth retardation. Amer. J. Dis. Child., 102: 249 (1961).
29. Winick, M.: Cellular growth in intrauterine malnutrition. Pediat. Clin. N. Amer., 17: 69 (1970).

30. Zamenhof, S., van Marthens, E., and Margolis, F. L.: DNA (cell number) and protein in neonatal brain: Alteration by maternal dietary protein restriction. Science, 160: 322 (1968).

31. Nippon Clea Co. Lid., Osaka, Japan.

32. Japan Isotope Corp., Tokyo, Japan.

33. Sakura Film Co. Lid., Osaka, Japan.

34. Requests for reprints should be addressed to: M. Shimada, M.D., Department of Pediatrics, Shiga Medical College, Seta, Otsu-shi 520-521 (Japan).

35. Received for publication July 10, 1976.

36. Accepted for publication October $27,1976$.
Asthma chemotaxis eczema
HLA-B12 neutrophil(s) skin infections

\title{
A Familial Defect of Neutrophil Chemotaxis with Asthma, Eczema, and Recurrent Skin Infections
}

\author{
JERRY C. JACOBS ${ }^{(28)}$ AND MICHAEL E. NORMAN \\ Departments of Pediatrics, College of Physicians and Surgeons, Columbia University, New York, and the University \\ of Pennsylvania, Philadelphia and Babies Hospital, the Children's Medical and Surgical Center, New York,
} New York, USA

\begin{abstract}
Summary
A defect in chemotaxis of peripheral blood polymorphonuclear leukocytes (PMN's) was demonstrated in both parents and three of four children in a single family afllicted with varying degrees of respiratory allergy, unusual onset of severe eczema in the first month of life, and recurrent bacterial skin infections. Of great interest was the identification of HLA-B12 at the B locus in all affected members but not in the unaffected child. The two children known since infancy to be most severely affected with eczema and recurrent infections are HLA identical and homozygous for HLA-B12. The child without eczema and infections had an intermediate cellular chemotactic defect most apparent on kinetic studies.
\end{abstract}

\section{Speculation}

Recently, several familial defects of neutrophil chemotaxis have been reported in association with syndromes of recurrent bacterial infections, reaginic hypersensitivity, and/or clinical atopy. Our studies extend these observations by describing the possible genetic relationships between atopy (e.g., eczema) chemotactic defects, and the HLA system. Future studies in this field should examine the association between chemotactic defects and the HLA system, and the requirements of both atopy and chemotactic defects to produce recurrent infections.

Migration of leukocytes to sites of inflammation and infection involves a number of complex interrelating processes which presently are poorly understood. However, each definition of a variant from normal, each "experiment of nature," may contribute to an increased understanding of these basic mechanisms and, ultimately, to more effective relief of symptoms.

Hill and Quie (6) first described a defect in neutrophil chemotaxis in three unrelated children with eczema, recurrent bacterial infections, and increased serum immunoglobulin E (IgE) levels. We now report our findings of a cellular chemotactic defect in the neutrophils of five of six family members, followed for 11 years and studied repeatedly during a 2.5-year period, who display a pattern of unusually severe chronic eczema, asthma, and recurrent bacterial skin infections, all starting in the first month of life. The repetitive pattern of onset of a severe dermatitis in the first month of life suggested the possibility of an immunologic deficiency. In this family, phenotypic expression of the anomaly is associated with the presence of the histocompatibility antigen HLA-B12.

\section{CASE REPORTS}

\section{CASE I (FW)}

The 46-year-old father of the four children reported below has suffered from asthma and severe eczema with lichenification all of his life. During childhood the eczema was frequently infected. He has been otherwise healthy.

CASE $2(D W)$

The 38-year-old mother of the four children reported below has suffered from asthma and mild atopic dermatitis all her life. She has been otherwise healthy. Her marriage to $F W$ is not consanguinous.

\section{CASE $3(A W)$}

This 16-year-old daughter of cases 1 and 2 has had eczema with frequent staphylococcal superinfection since early infancy. Her skin is greatly lichenified. At age 15 she had osteomyletis (presumed to be staphylococcal and treated as such) of the left tibia. Over the years she has had a number of hospitalizations for asthma, and many episodes of impetiginous superinfection of her eczema. Growth and development are normal.

CASE $4(R W)$

This 11-year-old son of cases 1 and 2 has had eczema and asthma since early infancy with recurrent episodes of impetigi- 
nous superinfection of the skin. The severe eczema has required the administration of prednisone, $10 \mathrm{mg}$, on alternate mornings on arising for the past 3 years. There have been a number of hospitalizations for asthma and recurrent need for treatment of staphylococcal skin infections. Developmental milestones were delayed but intelligence seems normal. Growth is normal.

\section{CASE 5 (EW)}

This 7-year-old daughter of cases 1 and 2 has had only mild asthma not requiring hospitalization at any time but has had eczema since early infancy with recurrent staphylococcal skin infections and impetigo. Her developmental milestones were also delayed, but she also seems of normal intelligence and has grown normally.

\section{CASE 6 (PW)}

This 12-year-old son of cases 1 and 2 has normal skin and has never had asthma. Developmental milestones were normal. He has ragweed allergy with seasonal rhinitis.

\section{MATERIALS AND METHODS}

\section{HUMORAL IMMUNITY}

Immunoglobulins $\mathrm{G}, \mathrm{A}$, and $\mathrm{M}$ were measured quantitatively on immunoplates (19). Immunoglobulin $E$ was measured by a radioimmunoassay technique (20). Specific IgE antibodies to ragweed antigen were measured by the radioallergosorbent test (21). $\mathrm{C}_{3}$ complement was measured by radial immunodiffusion (22), total hemolytic complement (CH50) by the method of Mayer (9). Skin testing for immediate hypersensitivity was carried out using a standard panel of antigens. Isohemagglutinins were measured by standard techniques. B-cell markers were measured using sheep erythrocytes (E), rabbit anti-sheep erythrocyte antibody $(A)$, and fresh mouse serum $(C)(3,8)$.

\section{CELLULAR IMMUNITY}

Skin tests for delayed hypersensitivity employed the following materials: (1) Candida extract (23), 100 protein-nitrogen units (PNU)/cc; (2) mumps (24); (3) streptokinase-streptodornase (25) $, 10,000$ units/cc for the former and 2500 units/cc for the latter, in a 1:10 dilution; (4) trichophytin (23), $500 \mathrm{PNU} / \mathrm{cc}$; and (5) tuberculin, first strength purified protein derivative. In all cases $0.1 \mathrm{ml}$ was injected intradermally and the degree of induration read at 24 and $48 \mathrm{hr}$.

Lymphocyte cultures employed phytohemagglutinin and pokeweed mitrogen to measure the proliferative response of the parents' peripheral blood lymphocytes (1). All cultures were performed in duplicate, with $1 \times 10^{6}$ cells/tube, cultured in $10 \%$ autologous plasma.

T-cell markers were assessed by the rosette technique, using sheep erythrocytes $(E)(3,8)$.

\section{INFLAMMATORY RESPONSE}

The cellular and humoral components of chemotaxis were studied by a previously described modification of the Boyden assay (14). A washed suspension of either control or patient PMN's at a final concentration of $5 \times 10^{6} / \mathrm{ml}$ was placed on one side of a 3 micropore size membrane. Materials with chemotactic activity were placed on the other side. These materials were generated from fresh control or patient plasma or serum by incubation with performed antigen-antibody complexes. After incubation for $3 \mathrm{hr}$ at $37^{\circ}$ the average number of PMN's per high power field reaching the lowermost surface was determined (10 contiguous high power fields were counted). All experiments were performed in duplicate, and most experiments were counted in "blind fashion" by a second observer. In several experiments, the procedure was modified to compare the migra- tion of control and patient PMN's at different incubation times (30-180 $\mathrm{min})$, and the effects of preincubating control PMN's in normal or patient serum. Family members received no drugs for a minimum of 1 week before study. In every experiment a normal control subject was studied on the same day as the patients. During the 2.5-year period that chemotaxis experiments were performed, 10 different normal control subjects were employed on different days. The mean chemotactic migration of control PMN's was $17.3 \pm 1.3$ when autologous serum was the source of chemotactic factors, and $17.9 \pm 1.4$ when autologous plasma was the source of chemotactic factors.

Atopic Patient Control Subjects. Eight unrelated children with chronic severe eczema and varying degrees of clinical asthma and/or allergic rhinitis were studied. All of these patients were receiving topical corticosteroid therapy; several were also receiving systemic antibiotics for bacterial infections.

Normal Control Subjects. Healthy adult laboratory volunteers were used as control subjects.

Random leukocyte mobility was measured by a modification of the method of Ketchel and Favour (13).

The cellular and humoral components of phagocytosis of baker's yeast particles were studied by the method of Miller and Nilsson (12). Opsonization and phagocytosis of Staphylococcus aureus was studied by the technique of Root and associates (16).

Leukocyte bactericidal activity using Staphylococcus aureus as the test organism was examined by the technique of Root and associates (16).

Inflammatory stimulation was studied by the method of Rebuck and Crowley, according to previously published techniques (14). Epinephrine stimulation of the marginal leukocyte pool, and hydrocortisone stimulation of the bone marrow leukocyte pool were studied by previously published techniques (14).

\section{HLA TYPING}

HLA typing was performed by the standard NIH two-stage microlymphocytotoxicity test.

\section{RESULTS}

\section{HUMORAL AND CELLULAR IMMUNITY, HLA TYPING}

The pertinent results of the studies of humoral and cellular immunity are presented in Figure 1, together with the family pedigree and results of HLA typing. IgE levels were markedly elevated in $A W, R W$, and $E W$ consistent with their history of atopy and severe eczema. The IgE level was normal in $P W$, who does not have eczema. Immediate hypersensitivity was noted in all family members to a variety of antigens, and peripheral eosinophilia was present in everyone but $F W$ and $P W$. Sensitivity to ragweed antigen at a single concentration $(10 \mathrm{PNU} / \mathrm{ml})$ was found by immediate skin testing in all family members, and appeared to correlate with RAST scores, but not with IgE levels. $P W$ had the most severe ragweed sensitivity, based on clinical symptomatology, skin tests, and RAST score.

IgG was mildly elevated in $A W$ and $R W$ and $\operatorname{IgA}$ was mildly elevated in $F W$. All other immunoglobulin levels and $\mathrm{C}_{3}$ and CH50 determinations were normal. Delayed hypersensitivity skin tests, in vitro lymphocyte proliferative responses, and numbers of circulating $\mathrm{T}$ - and $\mathrm{B}$-cells were in the normal range.

HLA typing revealed HLA-B12 at the B locus in all family members except $P W$. Two of the children, $A W$ and $R W$, were HLA identical and homozygous for HLA-B12.

\section{INFLAMMATORY RESPONSES}

With the exception of the chemotactic abnormality to be described, those humoral and cellular contributions to the inflammatory response that were tested were normal. 


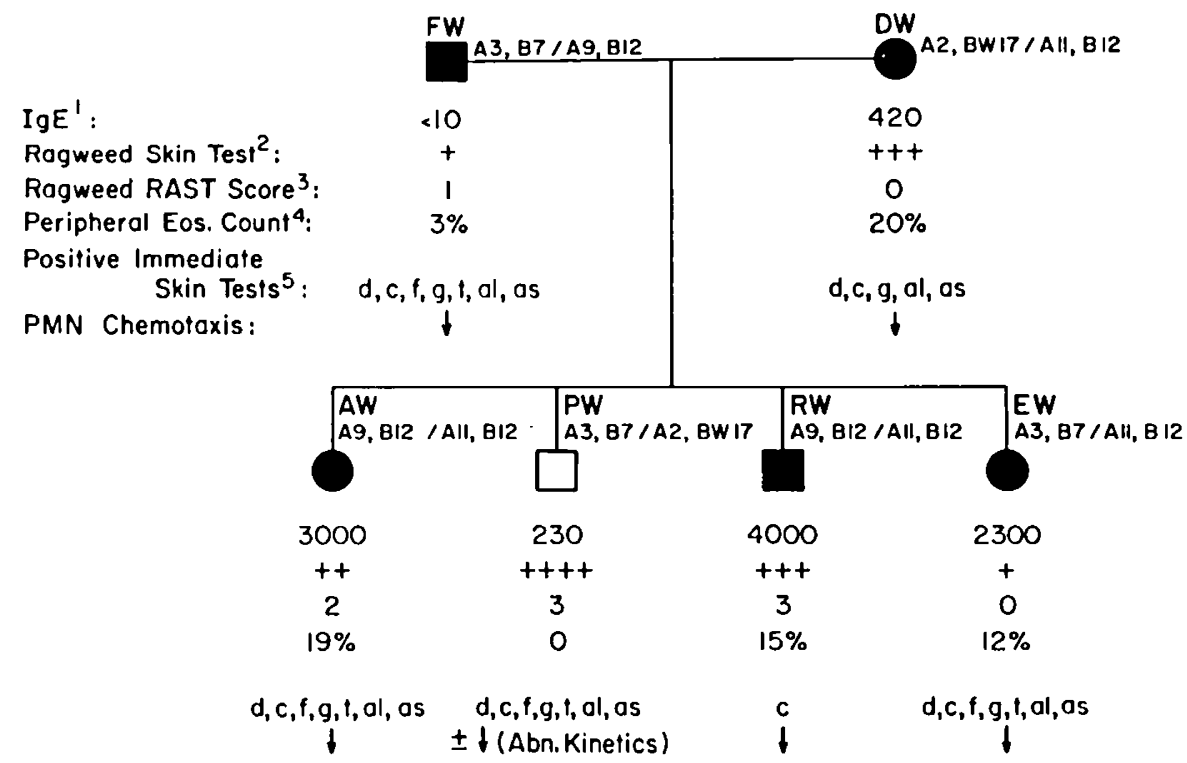

Fig. 1. Family pedigree together with the results of HLA typing and pertinent tests of cellular and humoral immunity. Closed symbols, eczema; open symbol, normal skin. ${ }^{1}$ : expressed in units per $\mathrm{ml}$ (normal adult 7-600). ${ }^{2}: 10 \mathrm{PNU} / \mathrm{ml} .{ }^{3}$ : explanation of RAST scores (score 0 , negative, no immunoglobulin E (IgE) antibodies; score 1, doubtful, insignificant levels of IgE antibodies; score 2, weakly positive, significant but low levels of IgE antibodies; score 3, moderately positive, moderate levels of $\mathrm{IgE}$ antibodies; score 4, strongly positive, very high levels of IgE antibodies. ${ }^{4}:$ expressed as percentage of total white blood cells. ${ }^{3}$ : $\mathrm{d}$, dust; c, cat; $\mathrm{f}$, feathers; $\mathrm{g}$, grass; $\mathrm{t}$, trees; al, Alternaria; as, Aspergillus; PMN: polymorphonuclear leukocytes.

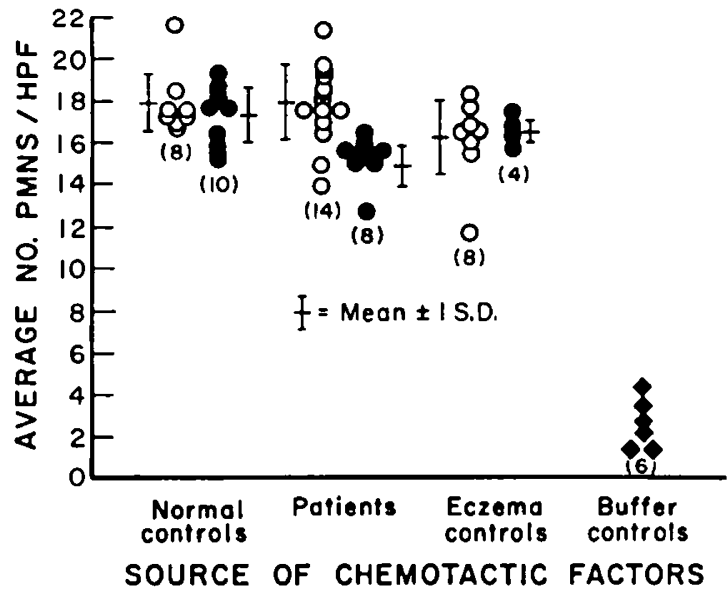

Fig. 2. The generation of chemotactic factors from the plasma and serum of normal control subjects, family members (patients), and eczema control subjects is compared. Normal polymorphonuclear leukocytes $(P M N S)$ were used for each set of experiments. Each point represents the mean of duplicate determinations and group mean values are indicated by the horizontal bars. $\bigcirc$ : plasma as a source of chemotactic factors; : serum as a source of chemotactic factors; $\$$ : buffer controls (no plasma or serum added to the bottom of the chambers). HPF: high power field.

\section{CHEMOTAXIS}

Studies of this family's plasma, serum, and cellular contributions to chemotaxis were performed repeatedly over a 2.5 -year period. On one occasion, eight atopic children with severe eczema served as control subjects. Results presented in Figure 2 compare group mean values of serum and plasma chemotactic factors from normal control subjects, eczema control subjects, and family members (nonpaired $t$-test). Each family member had serum and/or plasma chemotactic factors measured on at least four occasions, except $E W$ and $F W$, who were tested three times. Thus, there are 22 patient studies reported. There were no significant differences in the generation of plasma chemotactic

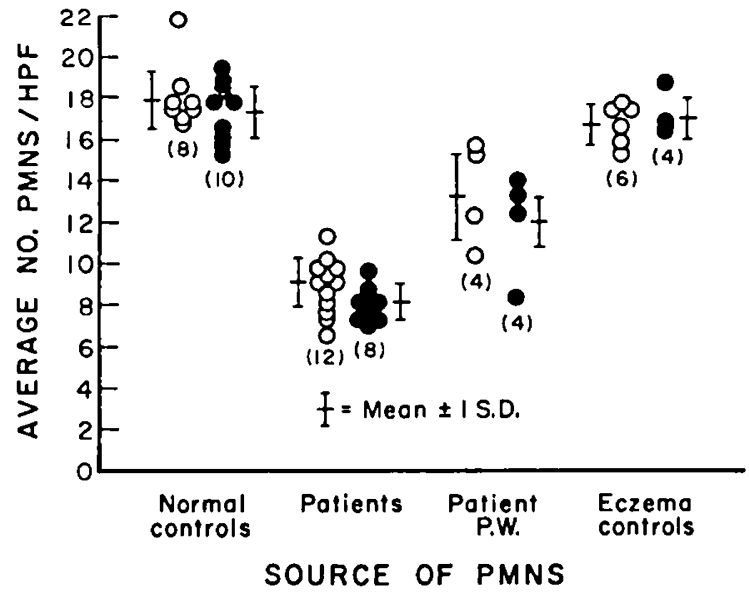

Fig. 3. The migration of polymorphonuclear leukocytes (PMNS) from normal control subjects, family members with eczema (patients), the unaffected family member $(P W)$, and eczema control subjects is compared. Normal plasma $(O)$ or serum $(\bullet)$ was used as a source of chemotactic factors in all of these experiments. Each point represents the mean of duplicate determinations and group mean values are indicated by the horizontal bars. HPF: high power field.

factors between normal control subjects $\chi=17.9, \mathrm{SD} \pm 1.4$ ), and the family members $(\bar{\chi}=17.9, \mathrm{SD} \pm 1.8)$ or eczema control subjects $(\bar{x}=16.2, \mathrm{SD} \pm 1.8)$. There was no significant difference in the generation of serum chemotactic factors between normal and eczema control subjects $(\bar{\chi}=17.3, \mathrm{SD} \pm 1.3$ versus $\bar{\chi}=16.5, \mathrm{SD} \pm 0.5)$, but a significant difference $(P<0.01)$ was demonstrated between normal control subjects and family members $(\bar{x}=17.3, \mathrm{SD} \pm 1.3$ versus $\bar{x}=15.3, \mathrm{SD} \pm 0.9)$.

In Figure 3, chemotactic migration of normal control PMN's was not significantly different from eczema control PMN's, using normal plasma as a source of chemotactic factors $(\bar{\chi}=17.9, \mathrm{SD}$ \pm 1.8 versus $\bar{\chi} 16.8, \mathrm{SD} \pm 1.0$ ), or normal serum as a source of chemotactic factors $(\bar{\chi}=17.3, \mathrm{SD} \pm 1.3$ versus $\bar{\chi}=17.1, \mathrm{SD} \pm$ 1.0). The migration of both normal and eczema control PMN's 
was strikingly different $(P<0.001)$ from patient PMN's, using normal plasma $(\bar{\chi}=9.1 \mathrm{SD} \pm 1.2)$ or normal serum $(\bar{\chi}=8.2$, $\mathrm{SD} \pm 0.9)$ as a source of chemotactic factors. In the single family member without eczema or infections $(P W)$ PMN migration was intermediate between the control subjects and all other affected family members, using either normal plasma $(\bar{\chi}=13.3, \mathrm{SD} \pm$ $2.1)$ or normal serum $(\bar{\chi}=12.1, \mathrm{SD} \pm 2.2)$ as a source of chemotactic factors. The differences between group means for $P W$ and control subjects were statistically significant $(0.001<P$ $<0.01)$. In these studies, the PMN's of all family members except $P W$ were tested on three separate occasions against normal plasma, normal serum, or both plasma and serum. PW's PMN's were tested four times.

Because of the differences in PMN migration of the affected family members and $P W$, a kinetic study was performed, comparing the migration of normal control PMN's, PMN's from $D W$ (with defect), and from $P W$ (intermediate defect). There was a linear relationship between chemotactic migration and time of incubation, up to $90 \mathrm{~min}$; no differences were noted in the degree of PMN chemotaxis (Fig. 4). From 90-180 min, however, $D W$ 's cells showed no further migration, whereas control and $P W$ cells continued to migrate.

Although not shown, mixing experiments between patient and normal control serum failed to demonstrate an inhibitor of chemotactic factor production of expression. Using control serum as a source of chemotactic factors, control cells displayed normal migration when preincubated in either control or patient serum, ruling out a cell-directed serum inhibitory factor as the cause of deficient PMN chemotaxis in the affected family members.

\section{DISCUSSION}

We have demonstrated a defect in the chemotaxis of peripheral blood PMN's of both parents and three of four children in a single family afflicted with varying degrees of respiratory allergy, unusual onset of eczema in the first month of life, and recurrent bacterial skin infections. We believe that this constitutes a unique syndrome in this family. The chemotactic defect has persisted over the 2.5-year period of repeated study, minimizing the possible contribution of day to day variations in chemotaxis to the observed defect. In the only member of the family without eczema or skin infections $(P W)$, PMN chemotaxis was intermediate between that demonstrated in the affected family members and the control subjects. In contrast to the findings in this family,

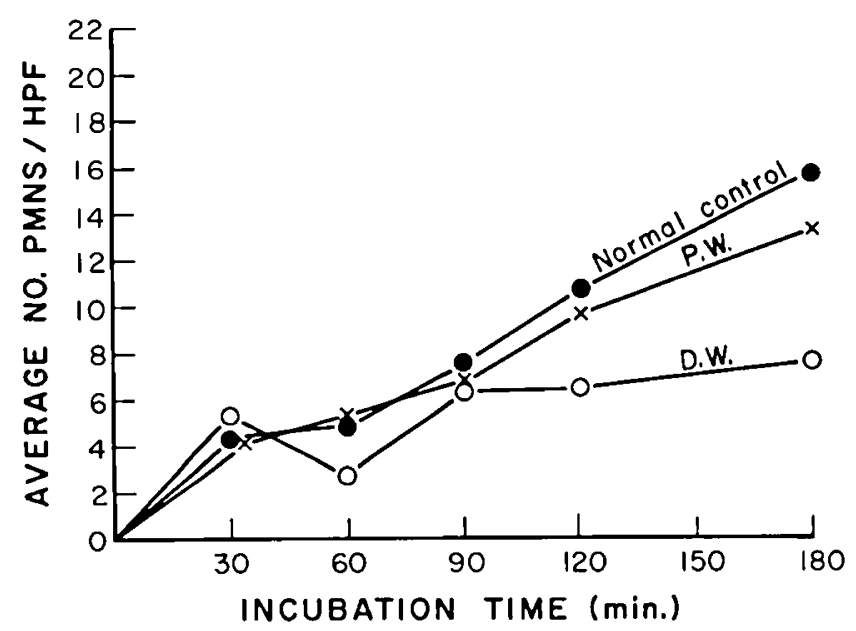

Fig. 4. The migration of polymorphonuclear leukocytes $(P M N S)$ from a control subject, an affected family member $(D W)$, and the unaffected family member $(P W)$ is compared during various incubation periods. A single normal serum specimen was used as the source of chemotactic factors. HPF: high power field. eight children with comparable degrees of atopic eczema and recurrent skin infections of varying degree demonstrated normal PMN chemotaxis. The inability of the affected family members' serum to inhibit chemotactic migration of control PMN's makes it unlikely that a cell-directed humoral inhibitor is the cause of the chemotactic defect. The difference in the chemotactic migration of $P W$ 's PMN's and those of his affected parents and three siblings was emphasized by the kinetic studies. Subtle but important differences in PMN migration may not be apparent unless kinetic or dose-response studies are performed in conjunction with standardized chemotactic assays (4).

Chemotactic factor production from immune complex activated family members' plasma was normal. Chemotactic factor production from activated family members' serum was slightly reduced when compared to control subjects. The reasons for this difference are not clear, but may be related to the smaller number of experiments performed with serum $(n=8)$ as compared to plasma $(n=14)$, and one markedly reduced value for serum chemotactic factor production (e.g., 13.2). The reduction in serum chemotactic factors could not be accounted for by complement deficiency, as $\mathrm{CH} 50$ levels were normal in all of the patients.

Of great interest was the identification of HLA-B12 at the B locus in all family members except $P W$. The two children known since infancy to be most severely affected with eczema and recurrent infections are HLA identical $(A W, R W)$ and homozygous for HLA-B12. These data, together with the clinical observations and chemotactic defect raises two important questions: $(I)$ is there an association between the inherited chemotactic defect and/or eczema and HLA-B12; (2) must the chemotactic defect and eczema occur together to produce the full expression of the syndrome?

If one hypothesizes that eczema is inherited as a dominant condition in this family and that both parents are heterozygous for this gene as well as for HLA-B12 (also a dominant gene), then the coincidence that $P W$ would not have eczema and not have HLA-B 12 would occur by chance with an incidence of 0.10 (lod score method). The probability of the combined coincidence of (1) three children with HLA-B12 having eczema; (2) two children homozygous for HLA-B12 having the most severe eczema; (3) one child heterozygous for HLA-B12 having less severe eczema; and (4) one child without HLA-B12 having no eczema occurring by chance is only 0.0078 . It is therefore possible that one determinant of this syndrome is a dominant gene for eczema, associated with the HLA-B12 locus. The chemotactic defect would then have to be inherited as a separate, dominant trait.

Since the chemotactic defect was fully expressed in all family members except $P W$, who has an intermediate chemotactic defect without eczema or HLA-B12, an alternative explanation must be considered; the chemotactic defect is inherited as a dominant trait and is itself linked to HLA-B12. For this to have occurred, however, would have required a recombination (crossover) on PW's sixth chromosome, since he has the chemotactic defect but no HLA-B12. The intermediate nature of the chemotactic defect might be explained by the absence of eczema and recurrent infections. It has been suggested by Hill and Quie (7) and other studies from our laboratory (15) that eczema may aggravate or enhance cellular chemotactic defects, perhaps through defects in the local inflammatory response. Although chromosomal recombination is well described in the mouse (2), it has not been studied in man. It seems more likely that the former hypothesis is correct, and that the full phenotypic expression of the syndrome requires at least the combination of eczema and a chemotactic defect. It is possible that other as yet unknown determinants associated with HLA-B12 are playing a role in this syndrome.

It is important to emphasize that our assumptions are based on a small family pedigree. However, the unavailability of other pertinent family members for study prevents a more thorough testing of these hypotheses. 
The association of various diseases with specific HLA antigens is well known, but must be distinguished from disorders that occur in families with a specific haplotype (5). Levine et al. (10) have reported the association of specific HLA haplotypes with ragweed pollen allergy in seven families, implying "close genetic linkage between the HLA system and a genetic locus controlling immune responsiveness to antigen E." In this family, however, no particular haplotype could be associated with clinical ragweed allergy or specific IgE antibody to antigen $E$.

On the basis of our data we are unable to relate hyperimmunoglobulinemia $\mathrm{E}$ per se to either eczema or the chemotactic defect. Patients with atopic eczema often have elevated IgE levels, but the converse is not the case. Defects in chemotaxis have been reported in relatively few individuals with eczema and increased IgE levels. All of our eight eczema control subjects had normal chemotaxis, but four out of eight had elevated $\mathrm{IgE}$ levels. Hill and Quie (7) have pointed out that not all patients with elevated $\operatorname{IgE}$ have defective chemotaxis.

There are many similarities in the clinical and laboratory findings between our patients and those reported by Hill and Quie (6), although their patients were unrelated. The clinical and laboratory evidence for reaginic hypersensitivity was substantial. Associated immunologic defects were not detected in either group of patients. The different features of our family were the familial nature of the chemotactic defect, possible association of symptoms with a distinct HLA antigen, and the identification of a partial or intermediate defect in the PMN's of $P W$, who does not have this antigen.

The defect present in this family is different from that recently discovered in another family and reported from this laboratory (13). Those patients suffered from congenital ichthyosis and recurrent tinea rubrum infections, clinical and laboratory evidence of atopy was absent, and the defect was present in only one parent. Two additional recent reports of familial chemotactic defects should be mentioned. Van Scoy et al. (17) described recurrent bacterial infections, chronic mucocutaneous candidiasis, and extreme hyperimmunoglobulinemia $\mathrm{E}$ in association with defective neutrophil chemotaxis and lymphocyte responses to Candida antigen in a mother and her daughter. Family studies suggested a familial pattern of hyperimmunoglobulinemia $E$ and defective chemotaxis. The mother but not the daughter had atopy. HLA typing was not performed. Witemeyer and Van Epps (18) described a neutrophil chemotactic defect in two of six siblings who also suffered from recurrent infections and were red headed. Although none of the three published family studies are strictly comparable to each other or to our own, it is clear that familial defects of neutrophil chemotaxis are of growing importance in delineating the causes of syndromes of recurrent infection afflicting multiple family members.

The link between in vitro defects of chemotaxis and recurrent bacterial infections remains a controversial and unresolved issue, in the absence of consistent in vivo correlates of leukotactic defects (11). In one of our affected patients $(D W)$, PMN counts, in vivo migration of PMN's (Rebuck window), and mobilization of PMN's from the bone marrow and marginal pools were normal. Nevertheless, the familial nature of this in vitro defect and its correlation with a distinct clinical syndrome strongly suggests a cause and effect relationship.

\section{CONCLUSION}

A familial defect of neutrophil chemotaxis is described affecting both parents and three of four children. The fourth child has a pattern of chemotactic migration intermediate between affected family members and control subjects. The complete chemotactic defect is associated with a distinct clinical syndrome of respiratory allergy, very early onset of eczema (e.g., first month of life), recurrent skin infections, and also with HLAB12. The fourth child is clinically well and does not have HLAB12. All other tests of immune function are normal in all family members. We postulate an association between two dominantly inherited genes (e.g., eczema and HLA-B12) and the separate inheritance of the chemotactic defect. However, full expression of the clinical syndrome requires the coexistence of eczema and the chemotactic defect.

\section{REFERENCES AND NOTES}

1. Abdou, N. I., and Abdou, N. L.: Bone marrow: The bursa equivalent in man? Science, 175: 446 (1972).

2. Benacerraf, B.: Genetic control of specific immune responses. In: Progress in Immunology, II, Vol. 2, p. 181 (Grune and Stratton, New York, 1975).

3. Bianco, C., Patrick, R., and Nussenweig, V.: A population of lymphocytes bearing a membrane receptor for antigen-antibody complement complexes. J. Exp. Med., 132: 702 (1970)

4. Clark, R. A., Root, R. K., Kimball, H. R., and Kirpatrick, C. H.: Defective neutrophil chemotaxis and cellular immunity in a child with recurrent infections. Ann. Intern. Med., 78: 515 (1973).

5. Dausset, J., Degos, L., and Hors, J.: The association of HLA antigens with disease. Clin. Immunol. Immunopathol., 3: 127 (1974)

6. Hill, H. R., and Quie, P. G.: Raised serum IgE levels and defective neutrophil chemotaxis in 3 children with eczema and recurrent bacterial infections. Lancet, $l$ : 183 (1974).

7. Hill, H. A., and Quie, P. G.: Defective neutrophil chemotaxis associated with hyperimmunoglobulinemia E. In: J. A. Bellanti and D. H. Dayton: The Phagocytic Cell in Host Resistance, pp. 249-260 (Raven Press, New York, 1975).

8. Jondal, M., Holm, G., and Wigzell, H.: Surface markers on human T and B lymphocytes: A large population of lymphocytes forming non-immune rosettes with sheep red blood cells. J. Exp. Med., 136: 207 (1972).

9. Kabat, E. A., and Mayer, M. M.: Experimental Immunochemistry, Ed. 2, Chap. 2 (Charles C Thomas, Publisher, Springfield, Ill., 1961).

10. Levine, B. B., Stember, R. H., and Fotino, M.: Ragweed hay fever: Genetic control and linkage to HLA haplotypes. Science, 178: 1201 (1972)

11. Miller, M. E.: Cell movement and host defenses. Ann. Intern. Med., 78: 601 (1973).

12. Miller, M. E., and Nilsson, U. R.: A familial defect of the phagocytosis enhancing activity of serum related to a dysfunction of the fifth component of complement (C5). N. Engl. J. Med., 282: 354 (1970).

13. Miller, M. E., Norman, M. E., Koblenzer, P. J., and Shonauer, T.: A new familial defect of neutrophil movement. J. Lab. Clin. Med., 82: 1 (1973).

14. Miller, M. E., Oski, F. A., and Harris, M. B.: Lazy-leukocyte syndrome: A new disorder of neutrophil function. Lancet, $1: 665$ (1971).

15. Paslin, D., and Norman, M. E.: Atopic dermatitis and impaired neutrophil chemotaxis in Job's syndrome. Arch. Dermatol. (In press.)

16. Root, R. K., Rosenthal, A. S., and Balestra, D. J.: Abnormal bactericidal, metabolic, and lysosomal functions of Chediak-Higashi syndrome leukocytes. J. Clin. Invest., 51: 649 (1972).

17. Van Scoy, R. E., Hill, H. R., Ritts, R. E., Jr., and Quie, P. G.: Familial neutrophil chemotaxis defect, recurrent bacterial infections, mucocutaneous candidiases, and hyperimmunoglobulinemia E. Ann. Intern. Med., 82: 766 (1975).

18. Witemeyer, S., and Van Epps, D. E.: A familial defect in cellular chemotaxis associated with red headedness and recurrent infection. J. Pediat., 89: 33 (1976).

19. Meloy Laboratories, Springfield, Va.

20. Phadebas, Pharmacia, Piscataway, N. J.

21. RAST, Pharmacia, Piscataway, N. J.

22. Hyland immunoplates, Travenol Corp., Costa Mesa, Calif.

23. Hollister-Stier, Spokane, Wash.

24. Lilly Co., Indianapolis, Ind.

25. Varidase, Lederle Laboratories, Pearl River, N. Y.

26. The authors wish to thank the nursing staff of the Clinical Research Center of the Hospital of the University of Pennsylvania for their expert assistance with the clinical studies, Dr. NAbih Abdou for performing the in vitro studies of cellular immunity, Dr. Howard Levene for statistical assistance, Dr. Richard Root for performing the phagocytic and leukocyte bactericidal assays, Dr. Chester Zmijewski for performing the HLA typing, Dr. Michael E. Miller for many helpful suggestions and review of the manuscript, and Ms. Arlene Taylor for technical assistance.

27. This research was supported by the Department of Health, Education and Welfare Public Health Service Grant 5MDI-RR40 and the John A. Hartford Foundation.

28. Requests for reprints should be addressed to: Jerry C. Jacobs, M.D., Babies Hospital, The Children's Medical and Surgical Center, 3969 Broadway, New York, N.Y. 10032 (USA).

29. Received for publication August 19, 1960.

30. Accepted for publication October 8, 1976. 